Pacific Journal of Mathematics

MINIMAL GERSCHGORIN SETS. II

Mater 


\title{
MINIMAL GERSCHGORIN SETS II
}

\author{
B. W. LeVINGER AND R. S. VARGA
}

The Gerschgorin Circle Theorem, which yields $n$ disks whose union contains all the eigenvalues of a given $n \times n$ matrix $A=\left(a_{i, j}\right)$, applies equally well to any matrix $B=\left(b_{i, j}\right)$ of the set $\Omega_{A}$ of $n \times n$ matrices with $b_{i, i}=a_{i, i}$ and $\left|b_{i, j}\right|=\left|a_{i, j}\right|$, $1 \leqq i, j \leqq n$. This union of $n$ disks thus bounds the entire spectrum $S\left(\Omega_{A}\right)$ of the matrices in $\Omega_{A}$. The main result of this paper is a precise characterization of $S\left(\Omega_{A}\right)$, which can be determined by extensions of the Gerschgorin Circle Theorem based only on the use of positive diagonal similarity transformations, permutation matrices, and their intersections.

Given any $n \times n$ complex matrix $A=\left(a_{i, j}\right)$, it is well known that the simplest of Gerschgorin arguments, which depends upon row sums of the moduli of off-diagonal entries of the matrix $X^{-1} A X, X$ a positive diagonal matrix, yields the union of $n$ disks which contains all the eigenvalues of $A$. It is clear that this union of $n$ disks necessarily contains all the eigenvalues of any $n \times n$ matrix in the set $\Omega_{A}$ defined as follows: $B=\left(b_{i, j}\right) \in \Omega_{A}$ if $b_{i, i}=a_{i, i}, 1 \leqq i \leqq n$, and $\left|b_{i, j}\right|=\left|a_{i, j}\right|$ for all $1 \leqq i, j \leqq n, i \neq j$. Hence, this union of $n$ Gerschgorin disks can be viewed as giving bounds for the entire spectrum $S\left(\Omega_{4}\right)=$ $\left\{z \mid \operatorname{det}(z I-B)=0\right.$ for some $\left.B \in \Omega_{4}\right\}$ of the set $\Omega_{\Delta}$.

It is logical to ask to what extent the spectrum $S\left(\Omega_{A}\right)$ can be more precisely determined by extensions of Gerschgorin's original argument [3]. In the previous paper [6], it was shown that

$$
\partial G\left(\Omega_{A}\right) \subset S\left(\Omega_{A}\right) \subset G\left(\Omega_{A}\right),
$$

where $G\left(\Omega_{A}\right)$ is the minimal Gerschgorin set deduced from $A$ and $\partial G\left(\Omega_{A}\right)$ is its boundary. The first inclusion of (1.1) states that every point of the boundary $\partial G\left(\Omega_{A}\right)$ of the minimal Gerschgorin set is then an eigenvalue of some $B \in \Omega_{\Delta}$. We now extend the results of [6] by making use of results of Schneider [4], and Camion and Hoffman [1]. In so doing, we shall precisely determine $S\left(\Omega_{4}\right)$.

To begin, let $P_{\phi}=\left(\delta_{i, \phi(j)}\right)$ be an $n \times n$ permutation matrix, where $\phi$ is a permutation of the integers $1 \leqq i \leqq n$ and $\delta_{i, j}$ is the Kronecker delta function, and let $X=\operatorname{diag}\left(x_{1}, x_{2}, \cdots, x_{n}\right)$, where $\boldsymbol{x}>\boldsymbol{0}$. Given $B \in \Omega_{A}$, we define the $n \times n$ matrix $M^{\phi}(\boldsymbol{x})$ by

$$
M^{\phi}(\boldsymbol{x})=\left(X^{-1} B X-\lambda I\right) P_{\phi}=\left(m_{i, j}\right),
$$

so that

Received May 19, 1964. 


$$
m_{i, j}=b_{i, \phi(j)} x_{\phi(j)} / x_{i}-\lambda \delta_{i, \phi(j)}, \quad 1 \leqq i, j \leqq n .
$$

Following Schneider [4], if $\lambda$ is an eigenvalue of $B$, then $M^{\phi}(\boldsymbol{x})$ is surely singular and thus not strictly diagonally dominant. Hence,

$$
\left|m_{i, i}\right| \leqq \sum_{j \neq i}\left|m_{i, j}\right|
$$

must be true for at least one $i, 1 \leqq i \leqq n$. Defining first

$$
\Lambda_{i}(\boldsymbol{x}) \equiv\left(\sum_{j \neq i}\left|a_{i, j}\right| x_{j}\right) / x_{i}, \quad 1 \leqq i \leqq n,
$$

then (1.4) implies that either

$$
\left|\lambda-a_{i, i}\right| \leqq \Lambda_{i}(\boldsymbol{x}) \text { if } \phi(i)=i,
$$

or

$$
2 x_{\phi(i)}\left|a_{i, \phi(i)}\right| / x_{i} \leqq\left|\lambda-a_{i, i}\right|+\Lambda_{i}(x) \text { if } \phi(i) \neq i .
$$

For any complex number $\sigma$, we consequently define

$$
r_{i}^{\phi}(\sigma ; \boldsymbol{x}) \equiv \Lambda_{i}(\boldsymbol{x})-\left|\sigma-a_{i, i}\right| \quad \text { if } \quad \phi(i)=i,
$$

and let

$$
r_{i}^{\phi}(\sigma ; \boldsymbol{x}) \equiv\left|\sigma-a_{i, i}\right|+\Lambda_{i}(\boldsymbol{x})-2\left|a_{i, \phi(i)}\right| x_{\phi(i)} \mid x_{i} \quad \text { if } \quad \phi(i) \neq i .
$$

With this, we next define the set $G_{i}^{\phi}(\boldsymbol{x})$ as

$$
G_{i}^{\phi}(\boldsymbol{x}) \equiv\left\{\sigma \mid r_{i}^{\phi}(\sigma ; \boldsymbol{x}) \geqq 0\right\}, \quad 1 \leqq i \leqq n .
$$

If $\phi(i)=i$, then $G_{i}^{\phi}(\boldsymbol{x})$ reduces to the familiar Gerschgorin disk $\left|z-a_{i, i}\right| \leqq \Lambda_{i}(\boldsymbol{x})$. If $\phi(i) \neq i$, we observe from $\left(1.7^{\prime}\right)$ that $G_{i}^{\phi}(\boldsymbol{x})$ is the closed exterior of a disk, and is thus an unbounded set.

Defining $G^{\phi}(\boldsymbol{x})$ to be the union of the sets $G_{i}^{\phi}(\boldsymbol{x})$ :

$$
G^{\phi}(\boldsymbol{x}) \equiv \bigcup_{i=1}^{n} G_{i}^{\phi}(\boldsymbol{x}),
$$

the inequalities of (1.6) and (1.6') show that if $\lambda \in S\left(\Omega_{A}\right)$, then $\lambda \in G_{i}^{\phi}(\boldsymbol{x})$ for some $i$, and hence $\lambda \in G^{\phi}(\boldsymbol{x})$. Thus, $S\left(\Omega_{A}\right) \subset G^{\phi}(\boldsymbol{x})$ for every $\boldsymbol{x}>\mathbf{0}$, and we then have that

$$
G^{\phi}\left(\Omega_{\Delta}\right) \equiv \bigcap_{\boldsymbol{x}>0} G^{\phi}(\boldsymbol{x}),
$$

called the minimal Gerschgorin set relative to the permutation $\phi$, is such that

$$
S\left(\Omega_{A}\right) \subset G^{\phi}\left(\Omega_{A}\right)
$$

for every permutation $\phi$. It is clear that $G^{\phi}\left(\Omega_{A}\right)$ is a closed set for 
any permutation $\phi$. Since $G_{i}^{\phi}(\boldsymbol{x})$ is a bounded set only when $\phi(i)=i$, it follows that $G^{\phi}\left(\Omega_{A}\right)$ is a bounded set only when $\phi$ is the identity permutation. We remark that the results of [6] are for the special case when $\phi$ is the identity permutation.

Since (1.11) is valid for any permutation $\phi$, it then follows that

$$
S\left(\Omega_{A}\right) \subset H\left(\Omega_{A}\right),
$$

where

$$
H\left(\Omega_{A}\right) \equiv \bigcap_{\phi} G^{\phi}\left(\Omega_{A}\right)
$$

In $\S 2$, we first characterize (Theorem 1 ) the minimal Gerschgorin sets $G^{\phi}\left(\Omega_{A}\right)$, and then show (Theorem 2) that their boundaries $\partial G^{\phi}\left(\Omega_{A}\right)$ are subsets of $S\left(\Omega_{4}\right)$. Finally, using a result of Camion and Hoffman [1], we prove (Theorem 3 ) in $\S 3$ our main result that

$$
S\left(\Omega_{A}\right)=H\left(\Omega_{A}\right) \text {. }
$$

Summarizing, the now elementary Gerschgorin Circle Theorem [3], applied to a particular matrix $A$, actually gives eigenvalue bounds for a set $\Omega_{A}$ of related matrices. Our main result is that the exact spectrum $S\left(\Omega_{A}\right)$ of $\Omega_{A}$ can be determined from extensions of the Gerschgorin Circle Theorem based only on positive diagonal similarity transformations, permutation matrices, and intersections.

In $\S 4$, we include an extension of a result of [6] concerning the number of eigenvalues of any $B \in \Omega_{A}$ in a bounded component of $G^{\phi}\left(\Omega_{A}\right)$. Finally, in $\S 5$ we include several examples to show how $S\left(\Omega_{A}\right)$ can be determined.

2. The Function $\nu_{\phi}(\sigma)$. In order to determine $G^{\phi}\left(\Omega_{A}\right)$, let $\sigma$ be any complex number, and consider the real $n \times n$ matrix $Q^{\phi}(\sigma)=\left(q_{i, j}\right)$ whose entries are defined by

$$
q_{i, j}=(-1)^{\delta_{i, j}}\left|a_{i, \phi(j)}-\sigma \delta_{i, \phi(j)}\right|, \quad 1 \leqq i, j \leqq n .
$$

Since the off-diagonal entries of $Q^{\phi}(\sigma)$ are nonnegative, then $Q^{\phi}(\sigma)$ is essentially nonnegative $[2 ; 5, \mathrm{p} .260]$, and hence we can associate with the matrix $Q^{\phi}(\sigma)$ the real number $\nu_{\phi}(\sigma)$, where $\nu_{\phi}(\sigma)$ is the (possibly multiple) eigenvalue of $Q^{\phi}(\sigma)$ with largest real part. From the Perron-Frobenius theory of nonnegative matrices [5, pp. 46-47], $\nu_{\phi}(\sigma)$ corresponds to a nonnegative eigenvector $\boldsymbol{y} \geqq \mathbf{0}$, i.e., $Q^{\phi}(\sigma) \boldsymbol{y}=$ $\nu_{\phi}(\sigma) \boldsymbol{y}$, and it is further known that

$$
\nu_{\phi}(\sigma)=\inf _{\boldsymbol{u}>0} \max _{1 \leqq i \leqq n}\left\{\frac{\left(Q^{\phi}(\sigma) \boldsymbol{u}\right)_{i}}{u_{i}}\right\} .
$$


We remark that $\nu_{\phi}(\sigma)$ is a continuous function of $\sigma$.

THEOREM 1. Let $A=\left(a_{i, j}\right)$ be an $n \times n$ complex matrix, let $\phi$ be any permutation, and let $\sigma$ be a complex number. Then, $\sigma \in G^{\phi}\left(\Omega_{4}\right)$ if and only if $\nu_{\phi}(\sigma) \geqq 0$.

Proof. From the definitions of $Q^{\phi}(\sigma)$ in $(2.1)$ and $r_{i}^{\phi}(\sigma ; \boldsymbol{x})$ in $(1.7)-$ $\left(1.7^{\prime}\right)$, it follows that

$$
r_{i}^{\phi}(\sigma ; \boldsymbol{x})=\left(\frac{x_{\phi(i)}}{x_{i}}\right)\left[\frac{\left(Q^{\phi}(\sigma) z\right)_{i}}{z_{i}}\right] \text {, where } z_{i} \equiv x_{\phi(i)} .
$$

Now, if $\sigma \in G^{\phi}\left(\Omega_{A}\right)$, then $\sigma \in G^{\phi}(\boldsymbol{x})$ for every $\boldsymbol{x}>\boldsymbol{0}$. But for every $\boldsymbol{x}>\boldsymbol{0}$, there is an $i$ such that $\sigma \in G_{i}^{\phi}(\boldsymbol{x})$, so that $r_{i}^{\phi}(\sigma ; \boldsymbol{x}) \geqq 0$. Since $\boldsymbol{x}>\mathbf{0}$, then $\left.\left(x_{\phi(i)}\right) x_{i}\right)$ is positive for all $1 \leqq i \leqq n$, and it therefore follows from (2.2) that

$$
\max _{1 \leqq i \leqq n}\left[\left(Q^{\phi}(\sigma) z\right)_{i} / z_{i}\right] \geqq 0 \quad \text { for every } \quad \boldsymbol{x}>0 .
$$

Clearly, as $\boldsymbol{x}>\boldsymbol{0}$ runs over all positive vectors, so does the corresponding vector $z>0$. Hence, $\nu_{\phi}(\sigma) \geqq 0$ from (2.2). Conversely, assume that $\nu_{\phi}(\sigma) \geqq 0$. From (2.2) and (2.3), it follows that $r_{i}^{\phi}(\sigma ; \boldsymbol{x}) \geqq 0$ for some $i$ for every $\boldsymbol{x}>0$. Hence, $\sigma \in G^{\phi}(\boldsymbol{x})$ for every $\boldsymbol{x}>\boldsymbol{0}$, and thus $\sigma \in G^{\phi}\left(\Omega_{A}\right)$, which completes the proof.

Our interest turns now to the boundary $\partial G^{\phi}\left(\Omega_{A}\right)$ of the minimal Gerschgorin set $G^{\phi}\left(\Omega_{A}\right)$. As usual, it is defined by

$$
\partial G^{\phi}\left(\Omega_{A}\right)=\overline{G^{\phi}\left(\Omega_{A}\right)} \cap \overline{G^{\phi}\left(\Omega_{A}\right)^{\prime}},
$$

where $\overline{G^{\phi}\left(\Omega_{A}\right)^{\prime}}$ is the closure of the complement $G^{\phi}\left(\Omega_{A}\right)^{\prime}$ of $G^{\phi}\left(\Omega_{A}\right)$. It follows from Theorem 1 that $G^{\phi}\left(\Omega_{A}\right)^{\prime}$ is the set of all $\sigma$ which satisfy $\nu_{\phi}(\sigma)<0$. Similarly, the boundary $\partial G^{\phi}\left(\Omega_{A}\right)$ of the minimal Gerschgorin set is the set of all $\sigma$ for which $\nu_{\phi}(\sigma)=0$, and to which there exists a sequence of complex numbers $\left\{z_{j}\right\}_{j=1}^{\infty}$ with $\lim _{j \rightarrow \infty} z_{j}=\sigma$ such that $\nu_{\phi}\left(z_{j}\right)<0$.

As in [6], we now show that every point of the boundary $\partial G^{\phi}\left(\Omega_{A}\right)$ is an eigenvalue of some matrix $B \in \Omega_{4}$.

Theorem 2. Let $A=\left(a_{i, j}\right)$ be an $n \times n$ complex matrix, and let $\phi$ be any permutation. If $\nu_{\phi}(\sigma)=0$, then $\sigma$ is an eigenvalue of some matrix $B \in \Omega_{A}$, and thus $\sigma \in S\left(\Omega_{A}\right)$.

Proof. If $\boldsymbol{\nu}_{\phi}(\sigma)=0$, then there exists a vector $\boldsymbol{y} \geqq \mathbf{0}$ with $\boldsymbol{y} \neq \mathbf{0}$ such that $Q^{\phi}(\sigma) \boldsymbol{y}=\mathbf{0}$. Writing $\quad\left(\sigma-a_{k, k}\right)=\left|\sigma-a_{k, k}\right| \exp \left(i_{\psi_{k}}\right)$, $1 \leqq k \leqq n$, let the $n \times n$ matrix $B=\left(b_{k, j}\right)$ be defined by 
(2.5) $b_{k, k}=a_{k, k} ; b_{k, j}=\left|a_{k, j}\right| \exp i\left\{\psi_{k}+\pi\left[-1+\delta_{k, \phi(k)}+\delta_{j, \phi(k)}\right]\right\}, k \neq j$.

It is evident that $B \in \Omega_{A}$, and if $y_{j}=z_{\phi(j)}$, it can be verified (upon considering separately the cases when $\phi(i)=i$ and $\phi(i) \neq i)$ that $Q^{\phi}(\sigma) \boldsymbol{y}=\mathbf{0}$ is equivalent to

$$
\sum_{j=1}^{n} b_{k, j} z_{j}=\sigma z_{k}, \quad 1 \leqq k \leqq n .
$$

Since $\boldsymbol{y} \neq \mathbf{0}$, then $\boldsymbol{z} \neq \mathbf{0}$, and we conclude from (2.6) that $\sigma$ is an eigenvalue of $B$, which completes the proof.

In order to prove a somewhat stronger result, let $\sigma \in \partial G^{\phi}\left(\Omega_{A}\right)$. Then, $\nu_{\phi}(\sigma)=0$ and $\sigma \in S\left(\Omega_{A}\right)$. But as $S\left(\Omega_{A}\right) \subset G^{\phi}\left(\Omega_{A}\right)$ from (1.11), we have the

CoRollary 1. Let $A$ be an $n \times n$ complex matrix. Then, for any permutation $\phi$,

$$
\partial G^{\phi}\left(\Omega_{A}\right) \subset \partial S\left(\Omega_{A}\right)
$$

In [6], an interesting geometrical property of the boundary $\partial G^{\phi}\left(\Omega_{A}\right)$ was given when $\phi$ was the identity permutation, and $A$ was assumed to be irreducible. In that case, each boundary point of $G^{\phi}\left(\Omega_{A}\right)$ was shown to be the intersection of $n$ Gerschgorin circles. An analogous result is true for an arbitrary permutation $\phi$, under slightly stronger hypotheses.

Corollary 2. Let $A$ be an $n \times n$ complex matrix, let $\phi$ be any permutation, and let $\sigma \in \partial G^{\phi}\left(\Omega_{A}\right)$. If $Q^{\phi}(\sigma)$ is irreducible, then there exists a vector $\boldsymbol{x}>\mathbf{0}$ such that $\sigma \in \partial G_{i}^{\phi}(\boldsymbol{x})$ for all $1 \leqq i \leqq n$.

Proof. If $Q^{\phi}(\sigma)$ is irreducible, then $Q^{\phi}(\sigma)$ is essentially positive [5, p. 257]. Thus, there exists a vector $z>0$ such that $Q^{\phi}(\sigma) z=$ $\nu_{\phi}(\sigma) z$. But, if $\sigma \in \partial G^{\phi}\left(\Omega_{A}\right)$, then $\nu_{\phi}(\sigma)=0$, and $Q^{\phi}(\sigma) z=\mathbf{0}$. Letting $\boldsymbol{x}>\boldsymbol{0}$ be defined component-wise by $z_{i}=x_{\phi(i)}$, it then follows from (2.3) that $r_{i}^{\phi}(\sigma ; \boldsymbol{x})=0$ for all $1 \leqq i \leqq n$. Now, $r_{i}^{\phi}(\sigma ; \boldsymbol{x})$ is obviously a continuous function of $\sigma$ from $(1.7)-\left(1.7^{\prime}\right)$, and from (1.8) we deduce that $\partial G_{i}^{\phi}(\boldsymbol{x})=\left\{\mu \mid r_{i}^{\phi}(\mu ; \boldsymbol{x})=0\right\}$. Hence, $\sigma \in \partial G_{i}^{\phi}(\boldsymbol{x})$ for all $1 \leqq i \leqq n$, which completes the proof.

We remark that is $\phi$ if the identity permutation, then $Q^{\phi}(\sigma)$ is irreducible for any $\sigma$ if and only if $A$ is irreducible. For general $\phi$, it is not difficult to show that $A$ irreducible implies that $Q^{\phi}(\sigma)$ is irreducible when $\sigma \neq a_{i, i}$ for any $i$.

3. Main Result. We shall now show that $S\left(\Omega_{A}\right)=H\left(\Omega_{A}\right) \equiv$ $\bigcap_{\phi} G^{\phi}\left(\Omega_{A}\right)$. Since $S\left(\Omega_{A}\right) \subset H\left(\Omega_{A}\right)$ by (1.12), it suffices to prove that 
$S\left(\Omega_{A}\right)^{\prime} \subset H\left(\Omega_{A}\right)^{\prime}$, where $S\left(\Omega_{A}\right)^{\prime}$ denotes the complement of $S\left(\Omega_{A}\right)$. This last inclusion will follow quite easily from the following theorem of Camion and Hoffman [1]:

Given an arbitrary $n \times n$ complex matrix $B=\left(b_{i, j}\right)$, let $\AA_{B}$ be the set of all matrices $C=\left(c_{i, j}\right)$ with $\left|c_{i, j}\right|=\left|b_{i, j}\right|$ for all $1 \leqq i, j \leqq n$. Then, if all matrices $C \in \stackrel{\Omega}{B}_{B}$ are nonsingular, there exists a positive diagonal matrix $X=\operatorname{diag}\left(x_{1}, \cdots, x_{n}\right), x_{i}>0$, and a permutation matrix $P_{\phi}=\left(\delta_{i, \phi(j)}\right)$ such that the matrix $M \equiv B X P_{\phi}=\left(m_{i, j}\right)$ is strictly diagonally dominant, i.e.,

$$
\left|m_{i, i}\right|>\sum_{j \neq i}\left|m_{i, j}\right| \text { for all } 1 \leqq i \leqq n .
$$

We first prove

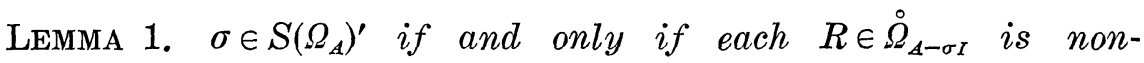
singular.

Proof. It is clear that each $R \in \stackrel{\Omega}{A-\sigma I}_{\text {. }}$ can be uniquely expressed as $R=D(B-\sigma I)$, where $D=\operatorname{diag}\left(e^{i \gamma_{1}}, \cdots, e^{i \psi_{n}}\right), \psi_{j}$ is real, and $B \in \Omega_{\Delta}$. Then, $\sigma \in S\left(\Omega_{A}\right)^{\prime}$ implies that $\operatorname{det}(B-\sigma I) \neq 0$ for any $B \in \Omega_{\Delta}$. But as $|\operatorname{det} D|=1$, then $\operatorname{det} R=\operatorname{det} D \cdot \operatorname{det}(B-\sigma I) \neq 0$ for any $R \in \AA_{\Delta}$. The converse follows similarly.

Now, suppose $\sigma \in S\left(\Omega_{A}\right)^{\prime}$. From Lemma 1 and the result of Camion and Hoffman applied to $B=A-\sigma I$, there exists a positive diagonal matrix $X=\operatorname{diag}\left(x_{1}, \cdots, x_{n}\right)$ and a permutation matrix $P_{\phi}=\left(\delta_{i, \phi(j)}\right)$ such that the matrix $M \equiv(A-\sigma I) X P_{\phi} \equiv\left(m_{i, j}\right)$ is strictly diagonally dominant, where

$$
m_{i, j}=\left(a_{i, \phi(j)}-\sigma \delta_{i, \phi(j)}\right) x_{\phi(j)} .
$$

Comparing (3.2) with the definition of $Q^{\phi}(\sigma)$ in (2.1) and setting $z_{j} \equiv$ $x_{\phi(j)}, 1 \leqq j \leqq n,(3.1)$ can be equivalently expressed as

$$
0>\sum_{j \neq i}\left|m_{i, j}\right|-\left|m_{i, i}\right|=\left(Q^{\phi}(\sigma) z\right)_{i}, \quad 1 \leqq i \leqq n .
$$

Since $z>0$, it follows from (2.2) that $\nu_{\phi}(\sigma)<0$, and hence from Theorem 1 we deduce that $\sigma \notin G^{\phi}\left(\Omega_{\Delta}\right)$. Consequently, $\sigma \notin S\left(\Omega_{\Delta}\right)$ implies that $\sigma \notin G^{\phi}\left(\Omega_{A}\right)$, which in turn implies that $\sigma \notin H\left(\Omega_{A}\right)$, or

$$
S\left(\Omega_{A}\right)^{\prime} \subset H\left(\Omega_{A}\right)^{\prime} \text {. }
$$

This, coupled with the result that $S\left(\Omega_{A}\right) \subset H\left(\Omega_{A}\right)$, gives us

THEOREM 3. Let $A=\left(a_{i, j}\right)$ be any $n \times n$ complex matrix. Then 
$S\left(\Omega_{A}\right)=H\left(\Omega_{A}\right)$

4. Disconnected minimal gerschgorin sets. A familiar result of Gerschgorin [3] states that if $k$ disks of the Gerschgorin set $G^{I}(\boldsymbol{x})$ (where $I$ is the identity permutation) are disjoint from the remaining $n-k$ disks, then these $k$ disks contain exactly $k$ eigenvalues of any matrix $B \in \Omega_{A}$. In this section, we give a generalization of this result (cf. Theorem 5 of [6]). For a given $n \times n$ matrix $A=\left(a_{i, j}\right)$ and an arbitrary permutation $\phi$, let $G_{j}^{\phi}\left(\Omega_{A}\right)$ denote the nonempty disjoint closed connected components of the minimal Gerschgorin set $G^{\phi}\left(\Omega_{A}\right)$ :

$$
G^{\phi}\left(\Omega_{A}\right)=\bigcup_{j=1}^{m} G_{j}^{\phi}\left(\Omega_{A}\right), \quad 1 \leqq m \leqq n .
$$

For each bounded component $G_{j}^{\phi}\left(\Omega_{A}\right)$, let the order $s_{j}^{\phi}$ be defined as the number of diagonal elements $a_{i, j}$ of $A$ contained in $G_{j}^{\phi}\left(\Omega_{A}\right)$ for which $\phi(i)=i$. We shall show that each matrix $B \in \Omega_{A}$ contains exactly $s_{j}^{\phi}$ eigenvalues in each bounded component $G_{j}^{\phi}\left(\Omega_{A}\right)$ of the minimal Gerschgorin set $G^{\phi}\left(\Omega_{A}\right)$.

To begin, we enlarge the set $\Omega_{A}$. An $n \times n$ matrix $B=\left(b_{i, j}\right)$ is defined to be an element of the extended set $\Omega_{A}^{\phi}$ if

$$
\left\{\begin{array}{l}
b_{i, i}=a_{i, i}, 1 \leqq i \leqq n ;\left|b_{i, \phi(i)}\right| \geqq\left|a_{i, \phi(i)}\right|, \phi(i) \neq i, \\
\left|b_{i, j}\right| \leqq\left|a_{i, j}\right|, 1 \leqq i, j \leqq n, \text { for which } j \neq i \text { and } j \neq \phi(i) .
\end{array}\right.
$$

Clearly, $\Omega_{\Delta} \subset \Omega_{A}^{\phi}$.

Lemma 2. Given $B \in \Omega_{A}^{\phi}$, then $G^{\phi}\left(\Omega_{B}\right) \subset G^{\phi}\left(\Omega_{A}\right)$.

Proof. For any vector $\boldsymbol{u}>\mathbf{0}$ and any complex number $\sigma$, consider the vector $Q_{B}^{\phi}(\sigma) u$, where we are using an obvious subscript notation. With $B \in \Omega_{A}^{\phi}$, one verifies from (4.2) and (2.1) that $Q_{B}^{\phi}(\sigma) \boldsymbol{u} \leqq Q_{A}^{\phi}(\sigma) \boldsymbol{u}$ for any $\boldsymbol{u}>\mathbf{0}$ and any $\sigma$, from which it follows that

$$
\max _{1 \leqq i \leqq n}\left\{\frac{\left(Q_{B}^{\phi}(\sigma) \boldsymbol{u}\right)_{i}}{u_{i}}\right\} \leqq \max _{1 \leqq i \leqq n}\left\{\frac{\left(Q_{A}^{\phi}(\sigma) \boldsymbol{u}\right)_{i}}{u_{i}}\right\} .
$$

Thus, from (2.2), $\nu_{\phi, B}(\sigma) \leqq \nu_{\phi, A}(\sigma)$. Hence, by Theorem $1, \sigma \in G^{\phi}\left(\Omega_{B}\right)$ implies that $\sigma \in G^{\phi}\left(\Omega_{A}\right)$, which completes the proof.

For this extended set $\Omega_{A}^{\phi}$, we remark that it can be further shown that $S\left(\Omega_{A}^{\phi}\right)=G^{\phi}\left(\Omega_{A}\right)$ for any permutation $\phi$. This generalizes another result (Theorem 6) of [6].

In the spirit of Gerschgorin's original continuity argument [3], we prove 
Theorem 4. Let $A=\left(a_{i, j}\right)$ be any $n \times n$ complex matrix, and let $\phi$ be any permutation. If $G^{\phi}\left(\Omega_{A}\right)$ has a bounded component $G_{j}^{\phi}\left(\Omega_{A}\right)$ of order $s_{j}^{\phi}$, then, for any matrix $B \in \Omega_{A}, B$ contains exactly $s_{j}^{\phi}$ eigenvalues in $G_{j}^{\phi}\left(\Omega_{A}\right)$.

Proof. For any $B=\left(b_{i, j}\right) \in \Omega_{\Delta}$, consider the family of matrices $B_{m}(\alpha)=\left(b_{i, j}(\alpha)\right)$ defined by

$$
\text { (4.4) }\left\{\begin{array}{l}
b_{i, i}(\alpha)=b_{i, i}, 1 \leqq i \leqq n \\
b_{i, \phi(i)}(\alpha)=b_{i, \phi(i)}[m(1-\alpha)+\alpha] \text { when } \phi(i) \neq i ; \\
b_{i, j}(\alpha)=\alpha b_{i, j} \text { for any } 1 \leqq i, j \leqq n \text { for which } j \neq i \text { and } j \neq \phi(i) .
\end{array}\right.
$$

By definition, $B_{m}(\alpha) \in \Omega_{A}^{\phi}$ for all $0 \leqq \alpha \leqq 1$ and all $m \geqq 1$, and $B_{m}(1)=B$. Moreover, $B_{m}(\alpha) \in \Omega_{B_{m}\left(\alpha^{\prime}\right)}^{\phi}$ for all $0 \leqq \alpha \leqq \alpha^{\prime} \leqq 1$. Thus, from Lemma 2, $G^{\phi}\left(\Omega_{B_{m}(\alpha)}\right) \subset G^{\phi}\left(\Omega_{A}\right)$ for all $0 \leqq \alpha \leqq 1$ and all $m \geqq 1$, and it is clear that the set $G^{\phi}\left(\Omega_{B_{m}(\alpha)}\right)$ increases monotonically with $\alpha$. We shall show that $B_{m}(0)$ has exactly $s_{j}^{\phi}$ eigenvalues in the bounded component $G_{j}^{\phi}\left(\Omega_{A}\right)$, and the theorem will follow by continuously increasing $\alpha$ from zero to unity.

From (4.4), the only possibly nonzero entries of the matrix $B_{m}(0)$ are $b_{i, i}(0)$ and $b_{i, \phi(i)}(0)$ where $\phi(i) \neq i$. Hence, by considering the disjoint cycles of the permutation $\phi$, we can find an $n \times n$ permutation matrix $P$ such that

$$
P B_{m}(0) P^{T}=\left[\begin{array}{ccc}
B_{1,1} & & 0 \\
B_{2,2} & \\
0 & \backslash & B_{N, N}
\end{array}\right], \quad 1 \leqq N<n .
$$

Here, $B_{1,1}$ is a diagonal matrix corresponding to all disjoint cycles with $\phi(i)=i$. The other matrices $B_{j, j}$ have the cyclic form

$$
B_{j, j}=\left[\begin{array}{ccc}
b_{1,1}^{(j)} & b_{1,2}^{(j)} & 0 \\
& 0 & b_{r_{j}-1, r_{j}}^{(j)} \\
b_{r_{j}, 1}^{(j)} & b_{r_{j}, r_{j}}^{(j)}
\end{array}\right], \quad 2 \leqq j \leqq N,
$$

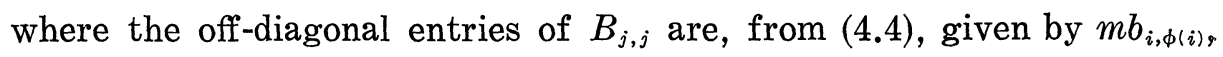
$\phi(i) \neq i$. Obviously, the eigenvalues of all the $B_{j, j}$ are the eigenvalues of $B_{m}(0)$.

The spectrum of matrices of the form (4.6) is discussed in Example 1 of the next section, and in $\S 6$ of [6]. We now assert that

$$
\left|b_{1,2}^{(j)} b_{2,3}^{(j)} \cdots b_{r_{j}, 1}^{(j)}\right| \neq 0 \text { for any } 2 \leqq j \leqq N .
$$

Otherwise, $b_{k, \phi(k)}=0$ for some integer $k$, where $\phi(k) \neq k$, and, as shown 
in the next section, this implies that $G^{\phi}\left(\Omega_{A}\right)$ is the entire complex plane. This contradicts the hypothesis that $G^{\phi}\left(\Omega_{4}\right)$ has a bounded component. From (4.4), we can write the product in (4.7) as $m^{r_{j}} \cdot K_{j}$, where $K_{j}$ is independent of $m$ and $\alpha$. Then, it is readily verified that the eigenvalues $\lambda$ of $B_{j, j}$ satisfy

$$
\prod_{k=1}^{r_{j}}\left|b_{k, k}^{(j)}-\lambda\right|=m^{r_{j}} \cdot K_{j}, \quad 2 \leqq j \leqq N,
$$

for any $B_{m}(0)$ derived from $B \in \Omega_{A}$. Since $B_{m}(0) \in \Omega_{A}^{\phi}$ for all $m \geqq 1$, we may choose $m$ to be arbitrarily large, and it is clear from (4.8) that the eigenvalues of $B_{j, j}$ must lie in an unbounded component of $G^{\phi}\left(\Omega_{A}\right)$ for any $2 \leqq j \leqq N$. Hence, the number of eigenvalues of $B_{m}(0)$ which lie in the bounded component $G_{j}^{\phi}\left(\Omega_{A}\right)$ is just the number of diagonal entries of $B_{1,1}$ in $G_{j}^{\phi}\left(\Omega_{A}\right)$, which by definition is precisely $s_{j}^{\phi}$. Now, increasing $\alpha$ continuously from zero to unity, it follows that $B$ has exactly $s_{j}^{\phi}$ eigenvalues in $G_{j}^{\phi}\left(\Omega_{A}\right)$, which completes the proof.

We remark that the order $s_{j}^{\phi}$ of a bounded component $G_{j}^{\phi}\left(\Omega_{A}\right)$ is a positive integer. For, if $s_{j}^{\phi}$ were zero, no $B \in \Omega_{\Delta}$ would have an eigenvalue in $G_{j}^{\phi}\left(\Omega_{A}\right)$, so that $S\left(\Omega_{A}\right) \cap G_{j}^{\phi}\left(\Omega_{A}\right)$ would be empty, which is a contradiction.

5. Some examples. We now give three examples to illustrate our results concerning the sets $S\left(\Omega_{4}\right), G^{\phi}\left(\Omega_{4}\right)$, and $H\left(\Omega_{4}\right)$.

EXAMPLE 1. It was previously shown [6] for the matrix

$$
A=\left[\begin{array}{cccc}
a_{1,1} & a_{1,2} & & 0 \\
0 & a_{2,2} & a_{2,3} & \\
& \searrow & \\
0 & & a_{n-1, n} \\
a_{n, 1} & & & a_{n, n}
\end{array}\right],
$$

where

$$
\left|a_{1,2} a_{2,3} \cdots a_{n, 1}\right|=1,
$$

that $\partial G^{I}\left(\Omega_{A}\right)=S\left(\Omega_{A}\right), I$ being the identity permutation. Let $\psi$ be the permutation $^{1}(123 \cdots n)$. If $\phi$ is any permutation other than $\psi$ or $I$, there is a positive integer $k, 1 \leqq k \leqq n$, such that $\phi(k) \neq k$, and $\phi(k) \neq \psi(k)$, so that $a_{k, \phi(k)}=0$. Thus, from $\left(1.7^{\prime}\right)$,

$$
r_{k}^{\phi}(\sigma ; \boldsymbol{x})=\left|\sigma-a_{k, k}\right|+\left|a_{k, \psi(k)}\right| x_{\psi(k)} / x_{k}>0
$$

for all $\boldsymbol{x}>\mathbf{0}$, and for all complex numbers $\sigma$. Hence, we deduce from

1 That is, in this section we are describing a permutation by its disjoint cycles. 
(2.2), (2.3), and Theorem 1 that $G^{\phi}\left(\Omega_{A}\right)$ is the entire complex plane. This argument shows more generally for an arbitrary matrix $A$ that any permutation $\phi$ which places a zero on the diagonal of $Q^{\phi}(\sigma)$ yields a minimal Gerschgorin set $G^{\phi}\left(\Omega_{A}\right)$ which is the entire complex plane.

For $\phi=I$, it was shown [6] for the matrix of (5.1) that

$$
G^{I}\left(\Omega_{A}\right)=\left\{\sigma\left|\prod_{i=1}^{n}\right| \sigma-a_{i, i} \mid \leqq 1\right\}
$$

and in an identical fashion, we can show that

$$
G \psi\left(\Omega_{A}\right)=\left\{\sigma\left|\prod_{i=1}^{n}\right| \sigma-a_{i, i} \mid \geqq 1\right\} .
$$

Hence, it follows that

$$
S\left(\Omega_{A}\right)=H\left(\Omega_{A}\right)=G^{I}\left(\Omega_{A}\right) \cap G^{\psi}\left(\Omega_{A}\right)=\partial G^{I}\left(\Omega_{A}\right) .
$$

ExAmPle 2. Consider the matrix

$$
A=\left[\begin{array}{lll}
2 & 0 & 1 \\
0 & 1 & 1 \\
1 & 1 & 2
\end{array}\right]
$$

In this case, there are only three permutations, corresponding to $\dot{\phi}=$ $I, \phi=(13)$, and $\phi=(23)$, for which $G^{\phi}\left(\Omega_{A}\right)$ is not the entire complex plane, and it is readily verified that

$$
\left\{\begin{array}{l}
G^{I}\left(\Omega_{A}\right)=\left\{\sigma|| 2-\left.\sigma\right|^{2} \cdot|1-\sigma| \leqq|1-\sigma|+|2-\sigma|\right\}, \\
G^{(13)}\left(\Omega_{A}\right)=\left\{\sigma|| 2-\left.\sigma\right|^{2} \cdot|1-\sigma| \geqq|1-\sigma|-|2-\sigma|\right\}, \\
G^{(23)}\left(\Omega_{A}\right)=\left\{\sigma|| 2-\left.\sigma\right|^{2} \cdot|1-\sigma| \geqq-|1-\sigma|+|2-\sigma|\right\} .
\end{array}\right.
$$

The boundaries $\partial G^{\phi}\left(\Omega_{A}\right)$ are obviously determined by choosing the equality signs in (5.7). The spectrum $S\left(\Omega_{A}\right)$ in this case is a multiply connected region and is illustrated in Figure 1.

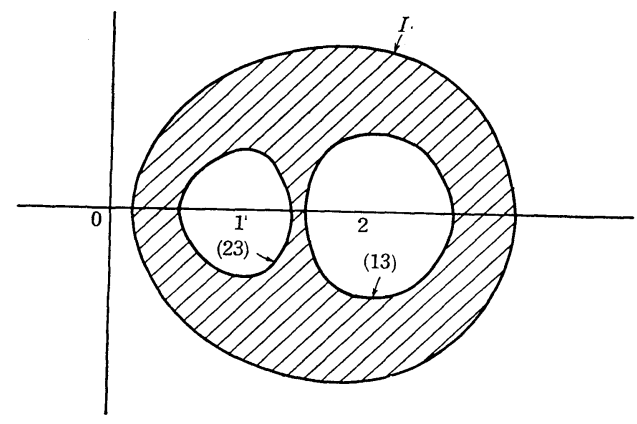

Fig. 1 
EXAMPle 3. Consider the matrix

$$
A=\left[\begin{array}{rrrr}
0 & 1 & 0 & 0 \\
0 & 0 & 1 & 0 \\
0 & 0 & 0 & 1 \\
-1 & -5 & -1 & -1
\end{array}\right]
$$

which is the companion matrix of the polynomial

$$
p_{4}(z)=z^{4}+z^{3}+z^{2}+5 z+1 .
$$

As previously shown, any permutation $\phi$ which places a zero on the diagonal of $Q^{\phi}(\sigma)$ yields a minimal Gerschgorin set $G^{\phi}\left(\Omega_{\alpha}\right)$ which is the entire complex plane. Consequently, we need consider only the permutations I, (1234), (234), and (34). The associated minimal Gerschgorin sets are given by

$$
\left\{\begin{array}{l}
G^{I}\left(\Omega_{A}\right)=\left\{\left.\sigma|| \sigma\right|^{3} \cdot|1+\sigma| \leqq 1+5|\sigma|+|\sigma|^{2}\right\}, \\
G^{(1234)}\left(\Omega_{A}\right)=\left\{\left.\sigma|| \sigma\right|^{3} \cdot|1+\sigma| \geqq 1-5|\sigma|-|\sigma|^{2}\right\}, \\
G^{(234)}\left(\Omega_{A}\right)=\left\{\left.\sigma|| \sigma\right|^{3} \cdot|1+\sigma| \geqq-1+5|\sigma|-|\sigma|^{2}\right\}, \\
G^{(34)}\left(\Omega_{A}\right)=\left\{\left.\sigma|| \sigma\right|^{3} \cdot|1+\sigma| \geqq-1-5|\sigma|+|\sigma|^{2}\right\} .
\end{array}\right.
$$

The last minimal Gerschgorin set $G^{(34)}\left(\Omega_{A}\right)$ is the entire complex plane, and thus yields no boundary components of $S\left(\Omega_{A}\right)$. The set $G^{(234)}\left(\Omega_{A}\right)$ yields, however, two separate boundaries, and $G^{(234)}\left(\Omega_{4}\right)$ has a bounded component. Applying Theorem 4, we can assert that each matrix of the set $\Omega_{A}$ has exactly one eigenvalue in this component, and hence each matrix of $\Omega_{\Delta}$ has exactly one eigenvalue in the inner annular region of Figure 2 .

These examples have interesting common features. In each ex-

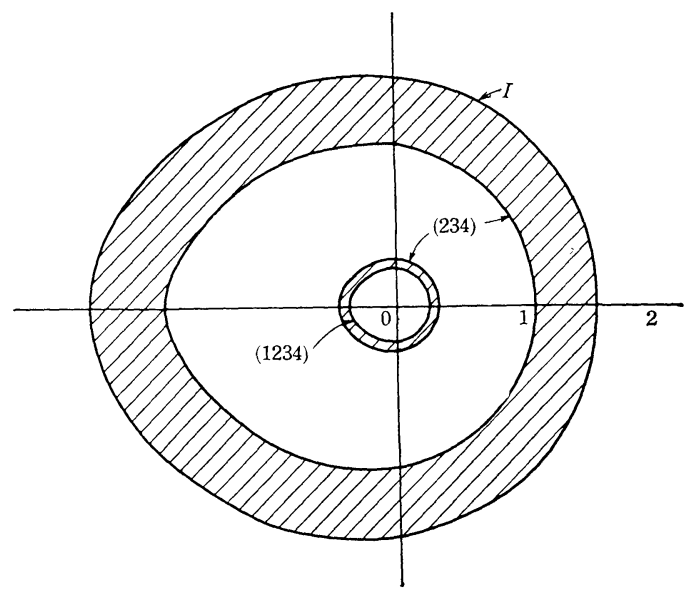

Fig. 2 
ample, the minimum number of permutations necessary to define all the boundary components of $S\left(\Omega_{A}\right)$ does not exceed the order $n$ of the matrix $A$. Similarly, the total number of boundary components of $S\left(\Omega_{A}\right)$ does not exceed $2 n$. We conjecture this to be true in general. We do point out that examples can be constructed where these upper bounds are attained.

\section{BIBLIOGRAPHY}

1. P. Camion and A. J. Hoffman, On the nonsingularity of complex matrices, Pacific J. Math. 17 (1966), 199-202.

2. Garrett Birkhoff and Richard S. Varga, Reactor criticality and non-negative matrices, J. Soc. Indust. Appl. Math. 6 (1958), 354-377.

3. S. Gerschgorin, Über die Abgrenzung der Eigenwerte einer Matrix, Izv. Akad. Nauk. SSSR, Ser. Mat. 7 (1931), 749-754.

4. Hans Schneider, Regions of exclusion for the latent roots of a matrix, Proc. Amer. Math. Soc. 5 (1954), 320-322.

5. Richard S. Varga, Matrix Iterative Analysis, Prentice-Hall, Inc. (1962), Englewood Cliffs, New Jersey.

6. Richard S. Varga, Minimal Gerschgorin sets, Pacific J. Math., 15 (1965), 719-729.

Case Institute of Technology 


\section{PACIFIC JOURNAL OF MATHEMATICS}

\section{EDITORS}

\author{
H. SAMELSON \\ Stanford University \\ Stanford, California \\ R. M. BLUMENTHAL \\ University of Washington \\ Seattle, Washington 98105
}

\author{
*J. DUGUNDJI \\ University of Southern California \\ Los Angeles, California 90007
}

RICHARD ARENS

University of California Los Angeles, California 90024

\section{ASSOCIATE EDITORS}
E. F. BECKENBACH
B. H. NEUMANN
F. WOLF
K. YoSIDA

\section{SUPPORTING INSTITUTIONS}

\author{
UNIVERSITY OF BRITISH COLUMBIA \\ CALIFORNIA INSTITUTE OF TECHNOLOGY \\ UNIVERSITY OF CALIFORNIA \\ MONTANA STATE UNIVERSITY \\ UNIVERSITY OF NEVADA \\ NEW MEXICO STATE UNIVERSITY \\ OREGON STATE UNIVERSITY \\ UNIVERSITY OF OREGON \\ OSAKA UNIVERSITY \\ UNIVERSITY OF SOUTHERN CALIFORNIA
}

\author{
STANFORD UNIVERSITY \\ UNIVERSITY OF TOKYO \\ UNIVERSITY OF UTAH \\ WASHINGTON STATE UNIVERSITY \\ UNIVERSITY OF WASHINGTON \\ * * * * \\ AMERICAN MATHEMATICAL SOCIETY \\ CHEVRON RESEARCH CORPORATION \\ TRW SYSTEMS \\ NAVAL ORDNANCE TEST STATION
}

Mathematical papers intended for publication in the Pacific Journal of Mathematics should be typewritten (double spaced). The first paragraph or two must be capable of being used separately as a synopsis of the entire paper. It should not contain references to the bibliography. Manuscripts may be sent to any one of the four editors. All other communications to the editors should be addressed to the managing editor, Richard Arens at the University of California, Los Angeles, California 90024.

50 reprints per author of each article are furnished free of charge; additional copies may be obtained at cost in multiples of 50 .

The Pacific Journal of Mathematics is published monthly. Effective with Volume 16 the price per volume (3 numbers) is $\$ 8.00$; single issues, $\$ 3.00$. Special price for current issues to individual faculty members of supporting institutions and to individual members of the American Mathematical Society: $\$ 4.00$ per volume; single issues $\$ 1.50$. Back numbers are available.

Subscriptions, orders for back numbers, and changes of address should be sent to Pacific Journal of Mathematics, 103 Highland Boulevard, Berkeley 8, California.

Printed at Kokusai Bunken Insatsusha (International Academic Printing Co., Ltd.), No. 6, 2-chome, Fujimi-cho, Chiyoda-ku, Tokyo, Japan.

\section{PUBLISHED BY PACIFIC JOURNAL OF MATHEMATICS, A NON-PROFIT CORPORATION}

The Supporting Institutions listed above contribute to the cost of publication of this Journal, but they are not owners or publishers and have no responsibility for its content or policies.

* Paul A. White, Acting Editor until J. Dugundji returns. 


\section{Pacific Journal of Mathematics}

\section{Vol. 17, No. 2 \\ February, 1966}

Henry A. Antosiewicz, Boundary value problems for nonlinear ordinary

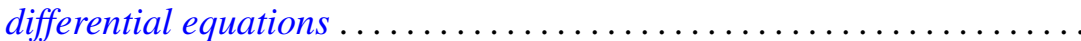

Bernard Werner Levinger and Richard Steven Varga, Minimal Gerschgorin sets. II ........................................

Paul Camion and Alan Jerome Hoffman, On the nonsingularity of complex matrices........................................ 211

J. Chidambaraswamy, Divisibility properties of certain factorials ........ 215

J. Chidambaraswamy, A problem complementary to a problem of Erdös .... 227

John Dauns, Chains of modules with completely reducible quotients ...... 235

Wallace E. Johnson, Existence of half-trajectories in prescribed regions and asymptotic orbital stability ............................ 243

Victor Klee, Paths on polyhedra. II . ........................ 249

Edwin Haena Mookini, Sufficient conditions for an optimal control problem in the calculus of variations ............................ 263

Zane Clinton Motteler, Existence theorems for certain quasi-linear elliptic equations........................................... 279

David Lewis Outcalt, Simple n-associative rings ............... 301

David Joseph Rodabaugh, Some new results on simple algebras ......... 311

Oscar S. Rothaus, Asymptotic properties of groups generation ........... 319

Ernest Edward Shult, Nilpotence of the commutator subgroup in groups admitting fixed point free operator groups .............

William Hall Sills, On absolutely continuous functions and the

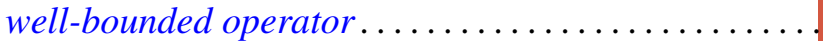

Joseph Gail Stampfli, Which weighted shifts are subnormal ..

Donald Reginald Traylor, Metrizability and completeness in normal Moore spaces 